\title{
Communication
}

\section{Laparoscopic Heated Intraperitoneal Chemotherapy in the Treatment of Carcinomatosis of Gastric Adenocarcinoma Origin}

\author{
Michael G. White (D) and Brian D. Badgwell * (D) \\ Department of Surgical Oncology, The University of Texas MD Anderson Cancer Center, \\ Houston, TX 77030, USA; mgwhite@mdanderson.org \\ * Correspondence: bbadgwell@mdanderson.org; Tel.: +1-(713)-745-7351
}

Citation: White, M.G.; Badgwell, B.D. Laparoscopic Heated Intraperitoneal Chemotherapy in the Treatment of Carcinomatosis of Gastric Adenocarcinoma Origin. J. Clin. Med. 2021, 10, 4757. https:// doi.org/10.3390/jcm10204757

Academic Editors: Masahiro Tajika and Santiago Gonzalez-Moreno

Received: 16 September 2021

Accepted: 15 October 2021

Published: 17 October 2021

Publisher's Note: MDPI stays neutral with regard to jurisdictional claims in published maps and institutional affiliations.

Copyright: (c) 2021 by the authors. Licensee MDPI, Basel, Switzerland. This article is an open access article distributed under the terms and conditions of the Creative Commons Attribution (CC BY) license (https:// creativecommons.org/licenses/by/ $4.0 /)$.

\begin{abstract}
The use of heated intraperitoneal chemotherapy (HIPEC) in conjunction with cytoreductive surgery has been gaining increasing traction in treating gastric adenocarcinoma with metastasis to the peritoneum in recent years. The addition of laparoscopic HIPEC (LS-HIPEC) to these treatment algorithms has increased the flexibility and adaptability of HIPEC integrating into treatment sequencing, allowing for iterative protocols of LS-HIPEC prior to cytoreduction as neoadjuvant treatment, as well as in the palliation of patients with unresectable disease and uncontrolled ascites. As the use of HIPEC in gastric adenocarcinoma continues to be refined, LS-HIPEC algorithms should continue to be considered and utilized both in curative treatment algorithms as well as in patients in the palliative setting. Given that LS-HIPEC remains a relatively nascent treatment modality, we advocate for its use in the setting of a clinical trial when feasible.
\end{abstract}

Keywords: carcinomatosis; HIPEC; gastric adenocarcinoma; regional therapies

The use of heated intraperitoneal chemotherapy (HIPEC) in managing carcinomatosis of gastric origin was first described by Fujimoto and colleagues in 1988 [1]. Since that time, these treatment algorithms have undergone significant study and refinement through a variety of observational studies and clinical trials. Nevertheless, the peritoneal spread of gastric adenocarcinoma is common, accounting for $49 \%$ of recurrences [2], and is associated with an overall poor prognosis despite modern systemic chemotherapy regimens with patients with M1 peritoneal disease surviving a median of 15 months [3]. Today these studies have resulted in improved patient selection, continued optimization of perfusate, the addition of minimally invasive techniques, and iterative neoadjuvant laparoscopic HIPEC (LS-HIPEC) protocols [4,5]. While primarily performed as a life-prolonging procedure, the low morbidity of LS-HIPEC has also allowed for its expanded use as a palliative procedure to control patients' symptomatic malignant ascites. In this monograph, we review the role of LS-HIPEC in the treatment of gastric cancer, including converting patients' disease from unresectable to resectable and palliating malignant ascites for patients receiving definitive systemic therapy. Finally, we discuss the early experience of pressurized intraperitoneal aerosol chemotherapy (PIPAC) as a recent additional tool in LS-HIPEC regimens [6].

As treatment algorithms defining the utility of HIPEC in the management of gastric cancer with peritoneal metastases began to become standardized in the 1990s, randomized control trials of cytoreductive surgery (CRS) for gastric cancer with and without HIPEC became feasible. The first of these randomized trials was completed by Yang et al., randomizing 68 patients to compare CRS (with gastrectomy) versus CRS + HIPEC with cisplatin and mitomycin C for 60-90 min via the coliseum technique. The addition of HIPEC demonstrated a statistically significant improvement in disease-specific survival from 6.5 to 11 months on uni- and multivariate analysis [7]. Moreover, this provided early subgroup analysis data demonstrating improved survival in those patients able to achieve an R- 0 or R-1 resection. This set the stage for the fundamental tenets of CRS and HIPEC in 
patients with isolated peritoneal gastric adenocarcinoma. Notably, HIPEC had the potential to improve survival, but this improvement required an R-0 or R-1 resection.

Reviews of non-randomized trials studying CRS and CRS HIPEC in the peritoneal spread of gastric cancer have demonstrated several CRS HIPEC algorithms that have reported variable median survivals ranging from 9 to 20 months [5,8-13]. Most recently, a retrospective case-control study (CYTO-CHIP) studied 277 patients in 19 French centers. This again demonstrated significantly improved overall survival in the CRS + HIPEC group (21.2 months) compared to those receiving CRS alone (14.7 months). Intriguingly the impact of the addition of HIPEC played a particularly strong role in decreasing rates of peritoneal recurrence in those patients with isolated peritoneal disease or peritoneal and extraperitoneal disease [14]. Meanwhile, treatment with HIPEC was most efficacious in the setting of poorly cohesive (PCC) gastric cancers with a median survival for PCC gastric cancer undergoing CRS + HIPEC of 34.5 months versus 14.3 months in the PCC CRS alone group [15].

While randomized trials of CRS with or without HIPEC ask an important and nuanced question, they do not address the more fundamental question of the benefit of operative intervention in these metastatic patients versus standard of care chemotherapy. This is a particularly difficult question to answer given the rarity of this disease and the rate at which systemic therapy options are added or refined. The GYMSSA trial attempted to answer this question, although they failed to fully accrue their target number of patients. Those that were accrued had metastatic gastric cancer (peritoneal or otherwise) treated with FOLFOXIRI and were compared to patients with peritoneal disease undergoing CRS and HIPEC. Although 4 of the 11 patients in the CRS/HIPEC group lived beyond 4 years, this came at the expense of $11 \%$ perioperative mortality. Along failing to meet accrual, this trial had a significant limitation in that many patients had received multiple lines of pretreatment prior to enrollment, and patients with liver or lung metastases were included in the systemic therapy arm as well [16].

Building on these experiences of open CRS HIPEC, the safety of iterative neoadjuvant LS-HIPEC in a western population was demonstrated in a phase II trial at our institution for patients with low-volume disease or positive cytology at the time of diagnosis. In this trial, patients with gastric adenocarcinoma and positive washings or low-volume peritoneal disease were enrolled and offered iterative neoadjuvant LS-HIPEC. They then underwent laparoscopic exploration, washings, and biopsy followed by perfusion with $30 \mathrm{mg}$ mitomycin C and $200 \mathrm{mg}$ cisplatin. This was repeated (up to 5 times) until patients cleared their positive cytology and/or biopsies and proceeded to resection, or their disease progressed. In cases of progression, patients proceeded to further systemic chemotherapy or another clinical trial [17]. This work showed iterative LS-HIPEC was safe and converted patients' disease from unresectable to resectable upon evaluation at the time of subsequent LS-HIPEC in 5/19 (26\%) cases [5]. Since that time, an additional phase I trial of a 3-drug regimen adding paclitaxel to mitomycin and cisplatin demonstrated this three-drug regimen to be safe. Expanded patient cohorts have allowed for the retrospective identification of prognostic factors, such as the presence of preoperative ascites or a high PCI score that are negatively associated with conversion to resectable disease in patients undergoing neoadjuvant LS-HIPEC protocols $[18,19]$. As such, our practice has used these and other studies to optimize contemporary treatment algorithms for our patients. Our current treatment algorithm is summarized in Figure 1.

In optimizing patient selection, it was noted that few patients with ascites at the time of initial laparoscopy were able to clear their disease and proceed to resection [19]. Currently, we advise patients with pretreatment ascites against enrollment in these protocols in the absence of extenuating circumstances and have opted to continue the use of two-drug oxaliplatin and mitomycin given observed equivalency with the three-drug regimen [19]. The safety of cytoreduction for gastric cancer and the use of LS-HIPEC in these patient populations has been demonstrated across countries, institutions, and a variety of treatment algorithms have been proposed. 


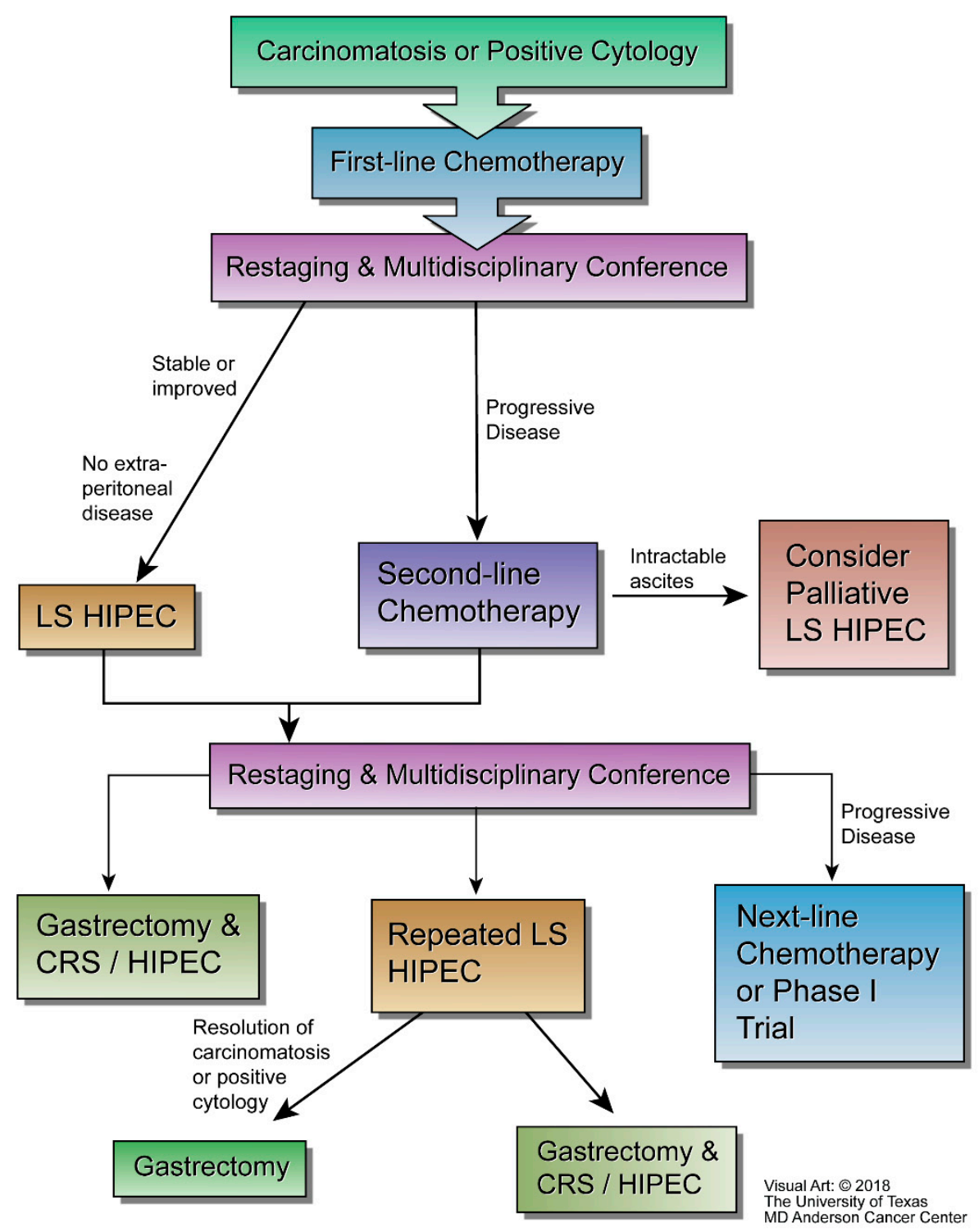

Figure 1. Flow chart of management of gastric cancer with carcinomatosis or positive cytology at MD Anderson Cancer Center.

Until recently, life-prolonging algorithms have been the primary focus of study in LS-HIPEC. At the same time, increasing interest in quality of life and symptom control has been considered in patients who did not qualify for or were not interested in these trials. For those patients with peritoneal spread of their gastric cancer, a common manifestation is symptomatic ascites. In order to palliate symptoms resulting from these ascites, several groups have performed laparoscopic peritoneal perfusion for patients with debilitating malignant ascites. The minimally invasive nature of this procedure offers minimal interruptions in systemic treatment regimens and minimizes medical risk in patients that are typically medically frail. In these cases, patients with unresectable disease and ascites undergoing definitive systemic treatment can undergo LS-HIPEC to decrease their ascitic burden and minimize the need for paracenteses. Although no randomized trials exist to study the use of LS-HIPEC in the treatment of malignant ascites, it has been favorably reported in several series, offering an option to palliate this difficult problem [20-22]. These studies demonstrate that a significant reduction in ascites can be achieved in $91-94 \%$ of patients in reported series [22,23]. While rates of significant complications (Grade 3 or 4) are low, even in these medically frail patients, ranging from 2 to $12 \%$ [22,24]. Here we have seen clear evidence of symptomatic relief in case series of patients whose treatment 
options are extremely limited and who are often highly symptomatic. Given the limited options for these patients, consideration should be given to this treatment modality at high volume centers familiar with LS-HIPEC after an in-depth discussion of the associated risks and benefits.

Recent years have seen further innovation in the use of pressurized intra-peritoneal aerosol chemotherapy (PIPAC) to treat unresectable gastric cancer. This treatment modality works by delivering pressurized and aerosolized chemotherapy as it travels through a laparoscopic port to maximize penetrance and delivery to the tissues. This route is thought to minimize adverse effects and is performed as a life-prolonging maneuver in patients with unresectable disease. These studies are currently performed in the setting of clinical trials in phase I [25,26], phase II [27-30], and early phase III [31] settings. These trials offer the potential for life prolongation while potentially improving quality of life over standard systemic chemotherapy options [32,33]. To date, data exclusively studying PIPAC in gastric cancer is limited, with reported radiologic response rates of 40\% [30] and histopathologic responses of 79\% [34]. Efficacy is difficult to interpret in small cohorts defining safety. Ultimately, further work is needed to define the role of this treatment modality in gastric cancer in the peritoneal metastatic setting, as well as define its utility in potentially curative treatment algorithms as neoadjuvant or adjuvant therapy.

While treatment pathways for patients with localized gastric cancer are clear and offer a potential for cure, those patients with peritoneal disease offer a more challenging gambit. In patients with low-volume disease with the potential to convert to a candidate for resection with LS-HIPEC, various algorithms have been defined as safe but have not been well studied in contrast to definitive systemic therapies with or without radiotherapy. We currently advocated for an iterative neoadjuvant two-drug treatment regimen in patients with good performance status who we expect will complete this regimen and would tolerate a potential eventual gastrectomy. To date, however, the utility of LS-HIPEC in prolonging survival in gastric cancer remains unknown. Retrospective comparisons at our institution have failed to demonstrate a survival benefit when comparing iterative LS-HIPEC to the standard of care chemotherapy. Experiences at other institutions and a meta-analysis of randomized clinical trials, however, have shown a marked and significant improvement [35-37]. Despite these contradictory findings, we and others observe a minority of patients who have an excellent and occasionally durable response. We, therefore, feel that further rigorous study is needed through prospective clinical trials such as the ongoing DRAGON II, in which randomized patients with locally advanced (T4) gastric cancer receive either an R0 resection and D2 lymphadenectomy followed by eight cycles of SOX (Doxorubicin, Adriamycin, and oxaliplatin with S-1) or neoadjuvant LS-HIPEC with Paclitaxel followed by three cycles SOX and R0 resection with D2 lymphadenectomy [38]. PERISCOPE trials randomizing patients with T3-T4 gastric cancer with limited peritoneal disease or positive cytology were randomized to palliative systemic chemotherapy or CRS + HIPEC after 3-4 cycles of systemic chemotherapy [39]. Additionally, we must prioritize translational research to better define disease biology and inform patient selection and the development of new agents and treatment modalities in the management of an aggressive malignancy such as gastric cancer.

Author Contributions: Conceptualization, M.G.W. and B.D.B.; writing-original draft preparation, M.G.W.; writing-review and editing, B.D.B. All authors have read and agreed to the published version of the manuscript.

Funding: This research received no external funding.

Institutional Review Board Statement: Not applicable.

Informed Consent Statement: Not applicable.

Data Availability Statement: No new data were created or analyzed in this study. Data sharing is not applicable to this article. 
Acknowledgments: MGW was supported by the National Institutes of Health (T32CA009599; MGW) and the MD Anderson Cancer Center support grant (P30 CA016672; MGW).

Conflicts of Interest: The authors declare no conflict of interest.

\section{References}

1. Fujimoto, S.; Shrestha, R.D.; Kokubun, M.; Ohta, M.; Takahashi, M.; Kobayashi, K.; Kiuchi, S.; Okui, K.; Miyoshi, T.; Arimizu, N.; et al. Intraperitoneal hyperthermic perfusion combined with surgery effective for gastric cancer patients with peritoneal seeding. Ann. Surg. 1988, 208, 36-41. [CrossRef]

2. Ikoma, N.; Chen, H.C.; Wang, X.; Blum, M.; Estrella, J.S.; Fournier, K.; Mansfield, P.; Ajani, J.; Badgwell, B.D. Patterns of Initial Recurrence in Gastric Adenocarcinoma in the Era of Preoperative Therapy. Ann. Surg. Oncol. 2017, 24, 2679-2687. [CrossRef]

3. Shiozaki, H.; Elimova, E.; Slack, R.S.; Chen, H.C.; Staerkel, G.A.; Sneige, N.; Shimodaira, Y.; Sagebiel, T.; Lee, J.H.; Bhutani, M.S.; et al. Prognosis of gastric adenocarcinoma patients with various burdens of peritoneal metastases. J. Surg. Oncol. 2016, 113, 29-35. [CrossRef] [PubMed]

4. Chang, E.; Alexander, H.R.; Libutti, S.K.; Hurst, R.; Zhai, S.; Figg, W.D.; Bartlett, D.L. Laparoscopic continuous hyperthermic peritoneal perfusion. J. Am. Coll. Surg. 2001, 193, 225-229. [CrossRef]

5. Badgwell, B.; Blum, M.; Das, P.; Estrella, J.; Wang, X.; Ho, L.; Fournier, K.; Royal, R.; Mansfield, P.; Ajani, J. Phase II Trial of Laparoscopic Hyperthermic Intraperitoneal Chemoperfusion for Peritoneal Carcinomatosis or Positive Peritoneal Cytology in Patients with Gastric Adenocarcinoma. Ann. Surg. Oncol. 2017, 24, 3338-3344. [CrossRef] [PubMed]

6. Nadiradze, G.; Giger-Pabst, U.; Zieren, J.; Strumberg, D.; Solass, W.; Reymond, M.A. Pressurized Intraperitoneal Aerosol Chemotherapy (PIPAC) with Low-Dose Cisplatin and Doxorubicin in Gastric Peritoneal Metastasis. J. Gastrointest. Surg. 2016, 20, 367-373. [CrossRef]

7. Yang, X.J.; Huang, C.Q.; Suo, T.; Mei, L.J.; Yang, G.L.; Cheng, F.L.; Zhou, Y.F.; Xiong, B.; Yonemura, Y.; Li, Y. Cytoreductive surgery and hyperthermic intraperitoneal chemotherapy improves survival of patients with peritoneal carcinomatosis from gastric cancer: Final results of a phase III randomized clinical trial. Ann. Surg. Oncol. 2011, 18, 1575-1581. [CrossRef] [PubMed]

8. $\quad$ Badgwell, B.; Ikoma, N.; Murphy, M.B.; Wang, X.; Estrella, J.; Roy-Chowdhuri, S.; Das, P.; Minsky, B.D.; Lano, E.; Song, S.; et al. A Phase II Trial of Cytoreduction, Gastrectomy, and Hyperthermic Intraperitoneal Perfusion with Chemotherapy for Patients with Gastric Cancer and Carcinomatosis or Positive Cytology. Ann. Surg. Oncol. 2021, 28, 258-264. [CrossRef] [PubMed]

9. Glehen, O.; Gilly, F.N.; Arvieux, C.; Cotte, E.; Boutitie, F.; Mansvelt, B.; Bereder, J.M.; Lorimier, G.; Quenet, F.; Elias, D.; et al. Peritoneal carcinomatosis from gastric cancer: A multi-institutional study of 159 patients treated by cytoreductive surgery combined with perioperative intraperitoneal chemotherapy. Ann. Surg. Oncol. 2010, 17, 2370-2377. [CrossRef]

10. Rau, B.; Brandl, A.; Piso, P.; Pelz, J.; Busch, P.; Demtroder, C.; Schule, S.; Schlitt, H.J.; Roitman, M.; Tepel, J.; et al. Peritoneal metastasis in gastric cancer: Results from the German database. Gastric Cancer 2020, 23, 11-22. [CrossRef]

11. Rihuete Caro, C.; Manzanedo, I.; Pereira, F.; Carrion-Alvarez, L.; Serrano, A.; Perez-Viejo, E. Cytoreductive surgery combined with hyperthermic intraperitoneal chemotherapy (HIPEC) in patients with gastric cancer and peritoneal carcinomatosis. Eur. J. Surg. Oncol. 2018, 44, 1805-1810. [CrossRef] [PubMed]

12. Yarema, R.; Mielko, J.; Fetsych, T.; Ohorchak, M.; Skorzewska, M.; Rawicz-Pruszynski, K.; Mashukov, A.; Maksimovsky, V.; Jastrzebski, T.; Polkowski, W.; et al. Hyperthermic intraperitoneal chemotherapy (HIPEC) in combined treatment of locally advanced and intraperitonealy disseminated gastric cancer: A retrospective cooperative Central-Eastern European study. Cancer Med. 2019, 8, 2877-2885. [CrossRef] [PubMed]

13. Van der Kaaij, R.T.; Wassenaar, E.C.E.; Koemans, W.J.; Sikorska, K.; Grootscholten, C.; Los, M.; Huitema, A.; Schellens, J.H.M.; Veenhof, A.; Hartemink, K.J.; et al. Treatment of PERItoneal disease in Stomach Cancer with cytOreductive surgery and hyperthermic intraPEritoneal chemotherapy: PERISCOPE I initial results. Br. J. Surg. 2020, 107, 1520-1528. [CrossRef] [PubMed]

14. Bonnot, P.E.; Piessen, G.; Kepenekian, V.; Decullier, E.; Pocard, M.; Meunier, B.; Bereder, J.M.; Abboud, K.; Marchal, F.; Quenet, F.; et al. Cytoreductive Surgery With or Without Hyperthermic Intraperitoneal Chemotherapy for Gastric Cancer With Peritoneal Metastases (CYTO-CHIP study): A Propensity Score Analysis. J. Clin. Oncol. 2019, 37, 2028-2040. [CrossRef] [PubMed]

15. Bonnot, P.E.; Lintis, A.; Mercier, F.; Benzerdjeb, N.; Passot, G.; Pocard, M.; Meunier, B.; Bereder, J.M.; Abboud, K.; Marchal, F.; et al. Prognosis of poorly cohesive gastric cancer after complete cytoreductive surgery with or without hyperthermic intraperitoneal chemotherapy (CYTO-CHIP study). Br. J. Surg. 2021. [CrossRef]

16. Rudloff, U.; Langan, R.C.; Mullinax, J.E.; Beane, J.D.; Steinberg, S.M.; Beresnev, T.; Webb, C.C.; Walker, M.; Toomey, M.A.; Schrump, D.; et al. Impact of maximal cytoreductive surgery plus regional heated intraperitoneal chemotherapy (HIPEC) on outcome of patients with peritoneal carcinomatosis of gastric origin: Results of the GYMSSA trial. J. Surg. Oncol. 2014, 110, 275-284. [CrossRef]

17. Badgwell, B.; Blum, M.; Das, P.; Estrella, J.; Wang, X.; Fournier, K.; Royal, R.; Mansfield, P.; Ajani, J. Lessons learned from a phase II clinical trial of laparoscopic HIPEC for gastric cancer. Surg. Endosc. 2018, 32, 512. [CrossRef]

18. Blum Murphy, M.; Ikoma, N.; Wang, X.; Estrella, J.; Roy-Chowdhuri, S.; Das, P.; Minsky, B.D.; Song, S.; Mansfield, P.; Ajani, J.; et al. Phase I Trial of Hyperthermic Intraperitoneal Chemoperfusion (HIPEC) with Cisplatin, Mitomycin, and Paclitaxel in Patients with Gastric Adenocarcinoma and Associated Carcinomatosis or Positive Cytology. Ann. Surg. Oncol. 2020, 27, 2806-2811. [CrossRef] 
19. White, M.G.; Kothari, A.; Ikoma, N.; Murphy, M.B.; Song, S.; Ajani, J.; Mansfield, P.; Badgwell, B. Factors Associated with Resection and Survival After Laparoscopic HIPEC for Peritoneal Gastric Cancer Metastasis. Ann. Surg. Oncol. 2020, 27, 4963-4969. [CrossRef] [PubMed]

20. Facchiano, E.; Scaringi, S.; Kianmanesh, R.; Sabate, J.M.; Castel, B.; Flamant, Y.; Coffin, B.; Msika, S. Laparoscopic hyperthermic intraperitoneal chemotherapy (HIPEC) for the treatment of malignant ascites secondary to unresectable peritoneal carcinomatosis from advanced gastric cancer. Eur. J. Surg. Oncol. 2008, 34, 154-158. [CrossRef]

21. Patriti, A.; Cavazzoni, E.; Graziosi, L.; Pisciaroli, A.; Luzi, D.; Gulla, N.; Donini, A. Successful palliation of malignant ascites from peritoneal mesothelioma by laparoscopic intraperitoneal hyperthermic chemotherapy. Surg. Laparosc. Endosc. Percutan. Tech. 2008, 18, 426-428. [CrossRef] [PubMed]

22. Valle, M.; van der Speeten, K.; Garofalo, A. Laparoscopic hyperthermic intraperitoneal peroperative chemotherapy (HIPEC) in the management of refractory malignant ascites: A multi-institutional retrospective analysis in 52 patients. J. Surg. Oncol. 2009, 100, 331-334. [CrossRef] [PubMed]

23. Jiao, J.; Li, C.; Yu, G.; Zhang, L.; Shi, X.; Yan, J.; Zhang, H.; Guo, P. Efficacy of hyperthermic intraperitoneal chemotherapy (HIPEC) in the management of malignant ascites. World J. Surg. Oncol. 2020, 18, 180. [CrossRef] [PubMed]

24. Valle, S.J.; Alzahrani, N.A.; Alzahrani, S.E.; Liauw, W.; Morris, D.L. Laparoscopic hyperthermic intraperitoneal chemotherapy (HIPEC) for refractory malignant ascites in patients unsuitable for cytoreductive surgery. Int. J. Surg. 2015, 23, 176-180. [CrossRef] [PubMed]

25. Raoof, M.; Malhotra, G.; Kohut, A.; O’Leary, M.; Frankel, P.; Tran, T.; Fakih, M.; Chao, J.; Lim, D.; Woo, Y.; et al. PIPAC for the Treatment of Gynecologic and Gastrointestinal Peritoneal Metastases: Technical and Logistic Considerations of a Phase 1 Trial. Ann. Surg. Oncol. 2021. [CrossRef] [PubMed]

26. Kim, G.; Tan, H.L.; Chen, E.; Teo, S.C.; Jang, C.J.M.; Ho, J.; Ang, Y.; Ngoi, N.Y.L.; Chee, C.E.; Lieske, B.; et al. Study protocol: Phase 1 dose escalating study of Pressurized Intra-Peritoneal Aerosol Chemotherapy (PIPAC) with oxaliplatin in peritoneal metastasis. Pleura Peritoneum 2018, 3, 20180118. [CrossRef] [PubMed]

27. Dumont, F.; Senellart, H.; Pein, F.; Campion, L.; Glehen, O.; Goere, D.; Pocard, M.; Thibaudeau, E. Phase I/II study of oxaliplatin dose escalation via a laparoscopic approach using pressurized aerosol intraperitoneal chemotherapy (PIPOX trial) for nonresectable peritoneal metastases of digestive cancers (stomach, small bowel and colorectal): Rationale and design. Pleura Peritoneum 2018, 3, 20180120. [CrossRef]

28. Eveno, C.; Jouvin, I.; Pocard, M. PIPAC EstoK 01: Pressurized IntraPeritoneal Aerosol Chemotherapy with cisplatin and doxorubicin (PIPAC C/D) in gastric peritoneal metastasis: A randomized and multicenter phase II study. Pleura Peritoneum 2018 3, 20180116. [CrossRef]

29. De Simone, M.; Vaira, M.; Argenziano, M.; Berchialla, P.; Pisacane, A.; Cinquegrana, A.; Cavalli, R.; Borsano, A.; Robella, M. Pressurized Intraperitoneal Aerosol Chemotherapy (PIPAC) with Oxaliplatin, Cisplatin, and Doxorubicin in Patients with Peritoneal Carcinomatosis: An Open-Label, Single-Arm, Phase II Clinical Trial. Biomedicines 2020, 8, 102. [CrossRef]

30. Struller, F.; Horvath, P.; Solass, W.; Weinreich, F.J.; Strumberg, D.; Kokkalis, M.K.; Fischer, I.; Meisner, C.; Konigsrainer, A.; Reymond, M.A. Pressurized intraperitoneal aerosol chemotherapy with low-dose cisplatin and doxorubicin (PIPAC C/D) in patients with gastric cancer and peritoneal metastasis: A phase II study. Ther. Adv. Med. Oncol. 2019, 11, 1758835919846402. [CrossRef]

31. Oliver Goetze, T.; Al-Batran, S.E.; Pabst, U.; Reymond, M.; Tempfer, C.; Bechstein, W.O.; Bankstahl, U.; Gockel, I.; Konigsrainer, A.; Kraus, T.; et al. Pressurized intraperitoneal aerosol chemotherapy (PIPAC) in combination with standard of care chemotherapy in primarily untreated chemo naive upper gi-adenocarcinomas with peritoneal seeding-a phase II/III trial of the AIO/CAOGI/ACO. Pleura Peritoneum 2018, 3, 20180113. [CrossRef] [PubMed]

32. Odendahl, K.; Solass, W.; Demtroder, C.; Giger-Pabst, U.; Zieren, J.; Tempfer, C.; Reymond, M.A. Quality of life of patients with end-stage peritoneal metastasis treated with Pressurized IntraPeritoneal Aerosol Chemotherapy (PIPAC). Eur. J. Surg. Oncol. 2015, 41, 1379-1385. [CrossRef]

33. Somashekhar, S.P.; Ashwin, K.R.; Rauthan, C.A.; Rohit, K.C. Randomized control trial comparing quality of life of patients with end-stage peritoneal metastasis treated with pressurized intraperitoneal aerosol chemotherapy (PIPAC) and intravenous chemotherapy. Pleura Peritoneum 2018, 3, 20180110. [CrossRef]

34. Gockel, I.; Jansen-Winkeln, B.; Haase, L.; Rhode, P.; Mehdorn, M.; Niebisch, S.; Moulla, Y.; Lyros, O.; Lordick, F.; Schierle, K.; et al. Pressurized Intraperitoneal Aerosol Chemotherapy (PIPAC) in Gastric Cancer Patients with Peritoneal Metastasis (PM): Results of a Single-Center Experience and Register Study. J. Gastric Cancer 2018, 18, 379-391. [CrossRef]

35. Blumenthaler, A.N.; Allen, C.J.; Ikoma, N.; Blum, M.; Das, P.; Minsky, B.D.; Mansfield, P.F.; Ajani, J.A.; Badgwell, B.D. Laparoscopic HIPEC for Low-Volume Peritoneal Metastasis in Gastric and Gastroesophageal Adenocarcinoma. Ann. Surg. Oncol. 2020, 27, 5047-5056. [CrossRef]

36. Yu, P.; Ye, Z.; Dai, G.; Zhang, Y.; Huang, L.; Du, Y.; Cheng, X. Neoadjuvant systemic and hyperthermic intraperitoneal chemotherapy combined with cytoreductive surgery for gastric cancer patients with limited peritoneal metastasis: A prospective cohort study. BMC Cancer 2020, 20, 1108. [CrossRef]

37. Granieri, S.; Bonomi, A.; Frassini, S.; Chierici, A.P.; Bruno, F.; Paleino, S.; Kusamura, S.; Germini, A.; Facciorusso, A.; Deraco, M.; et al. Prognostic impact of cytoreductive surgery (CRS) with hyperthermic intraperitoneal chemotherapy (HIPEC) in gastric cancer patients: A meta-analysis of randomized controlled trials. Eur. J. Surg. Oncol. 2021. [CrossRef] 
38. Beeharry, M.K.; Ni, Z.T.; Yang, Z.Y.; Zheng, Y.N.; Feng, R.H.; Liu, W.T.; Yan, C.; Yao, X.X.; Li, C.; Yan, M.; et al. Study protocol of a multicenter phase III randomized controlled trial investigating the efficiency of the combination of neoadjuvant chemotherapy (NAC) and neoadjuvant laparoscopic intraperitoneal hyperthermic chemotherapy (NLHIPEC) followed by R0 gastrectomy with intraoperative HIPEC for advanced gastric cancer (AGC): Dragon II trial. BMC Cancer 2020, 20, 224. [CrossRef]

39. Koemans, W.J.; van der Kaaij, R.T.; Boot, H.; Buffart, T.; Veenhof, A.; Hartemink, K.J.; Grootscholten, C.; Snaebjornsson, P.; Retel, V.P.; van Tinteren, H.; et al. Cytoreductive surgery and hyperthermic intraperitoneal chemotherapy versus palliative systemic chemotherapy in stomach cancer patients with peritoneal dissemination, the study protocol of a multicentre randomised controlled trial (PERISCOPE II). BMC Cancer 2019, 19, 420. [CrossRef] 